\title{
Exact exponents of edge singularities in dynamic correlation functions of 1D Bose gas
}

\author{
Adilet Imambekov and Leonid I. Glazman \\ Department of Physics, Yale University, New Haven, Connecticut, USA, 06520
}

(Dated: July 30, 2021)

\begin{abstract}
The spectral function and dynamic structure factor of bosons interacting by contact repulsion and confined to one dimension exhibit power-law singularities along the dispersion curves of the collective modes. We find the corresponding exponents exactly, by relating them to the known Bethe ansatz solution of the Lieb-Liniger model. Remarkably, the Luttinger liquid theory predictions for the exponents fail even at low energies, once the immediate vicinities of the edges are considered.
\end{abstract}

PACS numbers: $03.75 . \mathrm{Kk}$, 05.30.Jp, 02.30.Ik

In a typical experiment with cold atoms, the atomic ensemble is kept isolated from environment over certain time. This decoupling from the environmental degrees of freedom is effective enough to make the interaction between the atoms to be the leading cause of the ensemble's evolution in time. The isolation of ${ }^{87} \mathrm{Rb}$ atoms in elongated traps recently showed [1] the peculiarity of the dynamics of bosons confined to one dimension (1D), showing almost no relaxation, and consistent with the notion of the integrability.

Integrability allows one to find the spectrum of excitations of the quantum system by means of a thermodynamic Bethe ansatz (TBA), as it was done for the case of pointlike interaction between bosons by Lieb 2]. A more recent progress based on algebraic Bethe ansatz [3] ideas helped to develop sophisticated numerical methods for studying the dynamic responses of 1D Bose gas [4, 5]. However, in spite of more than 40 years of research, the analytic calculation of correlation functions from Bethe ansatz wave functions still remains a challenge. Some progress in understanding of dynamical correlations was achieved recently along a different path, which uses the effective Hamiltonian description and the physical analogy with the Fermi edge singularity [6, 7, [8, 9]. The analogy helped to show that the dynamic structure factor (DSF) and spectral function may exhibit power-law singularities along the dispersion curves of the collective modes. Very recently Pereira et al. [10] put forward an idea to combine the effective Hamiltonian method developed in Refs. [6, 7, [8] with the TBA method to study the edge singularities of XXZ spin chain. In their approach, Pereira et al. used the ideas based on conformal field theory to relate the parameters of the effective Hamiltonian to the finite-size corrections to the energy.

Here we find an alternative way to directly relate the parameters of the effective Hamiltonian to the scattering phase shifts found in the Bethe ansatz solution of the Lieb-Liniger model. We extend the formalism of Refs. [6, 7, 8] to evaluate the exponents not only of the DSF, but of the spectral function as well. In the low energy limit we analytically evaluate exact exponents as a function of Luttinger parameter $K$ only. Remarkably, the Luttinger liquid (LL) theory predictions [11, 12, 13, 14] for the exponents fail even at low energies, once the immediate vicinities of the edges are considered. Our results at low energies help understanding the behavior of $1 \mathrm{D}$ systems beyond the linear spectrum approximation of the LL theory. They can be used as a benchmark for numerical methods which attempt to evaluate many-body dynamics of continuous 1D models, e.g., using t-DMRG algorithms [15].

DSF describes the probability to excite the ground state with momentum and energy transfer $(k, \omega)$, and can be measured using Bragg spectroscopy [16]. Spectral function describes the tunneling probability for a particle (or a hole) with momentum and energy $(k, \omega)$ respectively. It can be measured using stimulated Raman transition combined with additional spin-flips [17, 18] .

In what follows we will be interested in the zerotemperature DSF

$$
S(k, \omega)=\int d x d t e^{i(\omega t-k x)}\langle\rho(x, t) \rho(0,0)\rangle,
$$

and spectral function $A(k, \omega)=-\frac{1}{\pi} \operatorname{Im} G(k, \omega) \operatorname{sign} \omega$, where Green's function $G(k, \omega)$ is defined by [19]

$$
G(k, \omega)=-i \iint d x d t e^{i(\omega t-k x)}\left\langle T\left(\Psi(x, t) \Psi^{\dagger}(0,0)\right)\right\rangle .
$$

Here $\Psi(x, t)$ and $\rho(x, t)$ are boson annihilation and density operators, and $T$ denotes time ordering. Energy $\omega$ is measured from chemical potential, so $A(k, \omega)$ for $\omega>0(\omega<0)$ describes the response of the system to an addition of an extra particle (hole). Both $A(k, \omega)$ and $S(k, \omega)$ do not change under transformation $k \rightarrow-k$, and we will consider them only for $k>0$. Also $S(k, \omega)=0$ for $\omega<0$, so we consider DSF only for $\omega>0$.

The exactly solvable Lieb-Liniger model [2, 3] is defined by

$$
H_{L L}=-\sum_{j=1}^{N} \frac{\partial^{2}}{\partial z_{j}^{2}}+2 c \sum_{1 \leq j<k \leq N} \delta\left(z_{j}-z_{k}\right)-h N .
$$

Here $N$ is the total number of particles, $h$ is the chemical potential, $c>0$ is the interaction strength. Hereinafter we set $\hbar=1$ and the mass equals $1 / 2$ for brevity. The 
$\omega$

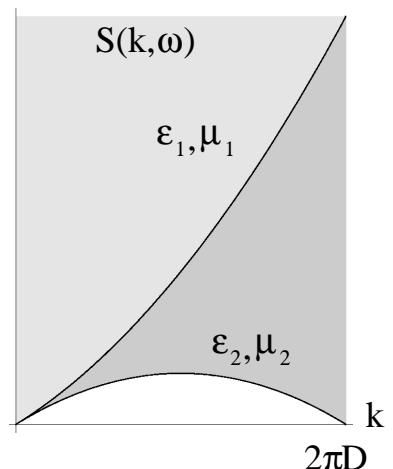

$\omega$

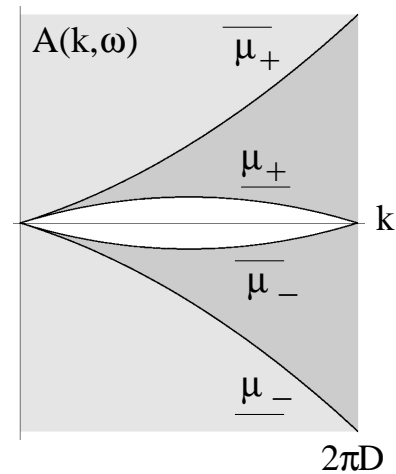

FIG. 1: (a) Dynamic structure factor (DSF) $S(k, \omega)$ and (b) spectral function $A(k, \omega)$. Shaded areas indicate the regions where they are nonvanishing. Lieb's particle mode $\varepsilon_{1}(k)$ and hole excitation mode $\varepsilon_{2}(k)$ are indicated. For spectral function $A(k, \omega)$ region with $\omega>0(\omega<0)$ corresponds to the particle (hole) part of the spectrum. Notations of $\mu$ indicate which exponents of Eqs. (16)-(18) should be used in Eq. (4).

ground state is fully characterized by the dimensionless parameter $\gamma=c / D$, where $D=N / L$ is the density. The regime of weak interactions corresponds to $\gamma \ll 1$, while the strong interactions (Tonks-Girardeau limit) correspond to $\gamma \gg 1$. An important parameter appearing in the effective hydrodynamic description [11, 12, 13] of the model (3) is the LL parameter $K=v_{f} / v$, where $v$ is the sound velocity and $v_{f}=2 \pi D$ is the Fermi velocity of noninteracting Fermi gas of density $D$. Parameter $K>1$ is uniquely defined by $\gamma$, with $K \approx \pi \gamma^{-1 / 2}$ for $\gamma \ll 1$ and $K \approx 1+4 / \gamma$ for $\gamma \gg 1$ [2, 13]. Energies of excitations can be also obtained, and give rise to Lieb's particle $\left(\varepsilon_{1}\right)$ and hole $\left(\varepsilon_{2}\right)$ excitations, as shown in Fig,1. The minimal excitation energy (measured from the ground state) of a state with total momentum $k$ equals $\varepsilon_{2}(k)$, and both $A(k, \omega)$ and $S(k, \omega)$ vanish identically for $|\omega|<\varepsilon_{2}(k)$.

At zero temperature, $S(k, \omega)$ and $A(k, \omega)$ have powerlaw behavior [ [6, 7,, 8] near $\pm \varepsilon_{1(2)}(k)$,

$$
S(k, \omega), A(k, \omega) \sim \text { const }+\left|\frac{1}{\omega \pm \varepsilon_{1(2)}(k)}\right|^{\mu},
$$

with notations of $\mu$ shown in Fig. 1 We provide exact results for exponents at $\pm \varepsilon_{1}(k)$ with $k>0$, and for exponents at $\pm \varepsilon_{2}(k)$ with $0<k<2 \pi D$, see Eqs. (16) - (26).

Let us briefly review the solution of the Lieb-Liniger model to set the notations, and we will mostly follow the conventions of Ref. [3]. Ground state quasimomenta $\nu_{j}, 1 \leq j \leq N$, are given by the solutions of Bethe equations

$$
L \nu_{j}+\sum_{k=1}^{N} \theta\left(\nu_{j}-\nu_{k}\right)=2 \pi n_{j},
$$

where $\theta(x)=2 \arctan \frac{x}{c}$ is the two-particle phase shift and quantum numbers are $n_{j}=j-1-(N-1) / 2$. In the thermodynamic limit, this system gives rise to the integral equation

$$
2 \pi \rho(\nu)-\int_{-q}^{q} K(\nu, \mu) \rho(\mu) d \mu=1 .
$$

Here $\rho(\nu)=\lim 1 /\left(L\left(\nu_{k+1}-\nu_{k}\right)\right)$ is the density of roots, $K(\nu, \mu)=\frac{2 c}{c^{2}+(\nu-\mu)^{2}}$, and $\pm q$ is the highest (lowest) filled quasimomentum; $q$ is defined as a function of density by the normalization condition $D=\int_{-q}^{q} \rho(\nu) d \nu$. Particlelike excitations with $k>0$ can be constructed by adding an extra quasimomentum $\lambda>q$, while holelike excitations are obtained by removing a quasimomentum $|\lambda|<q$ (particlelike states with $k<0$ correspond to $\lambda<-q$ ). Since all quasimomenta $\nu_{j}$ are coupled to each other by Eqs. (5), this will shift all of them. A convenient way to take this change into account is to introduce a shift function

$$
F_{B}(\nu \mid \lambda)= \pm\left(\nu_{j}-\tilde{\nu}_{j}\right) /\left(\nu_{j+1}-\nu_{j}\right),
$$

where $\tilde{\nu}_{j}$ are new solutions and upper (lower) sign corresponds to extra particle (hole). In the thermodynamic limit $F_{B}(\nu \mid \lambda)$ satisfies an integral equation [20]

$$
F_{B}(\nu \mid \lambda)-\frac{1}{2 \pi} \int_{-q}^{q} K(\nu, \mu) F_{B}(\mu \mid \lambda) d \mu=\frac{\pi+\theta(\nu-\lambda)}{2 \pi} .
$$

Shift function $F_{B}(\nu \mid \lambda)$ can be used [3] to calculate exact energies $\varepsilon_{1(2)}$ as a function of momentum $k(\lambda)$. The latter can be also written [3] as

$$
k(\lambda)= \pm\left(\lambda-\pi D+\int_{-q}^{q} \theta(\lambda-\nu) \rho(\nu) d \nu\right) .
$$

Here upper (lower) sign corresponds to particle (hole) excitation with $\lambda>q(|\lambda|<q)$, and $k(q)=0, k(-q+$ $0)=2 \pi D$.

As will be shown below, $F_{B}( \pm q \mid \lambda)$ play a crucial role in the calculation of the edge singularities, so we will investigate it in more detail. One can analytically derive the limiting behavior

$$
F_{B}( \pm q \mid q)=1 / 2 \pm(1 / 2-1 /(2 \sqrt{K}))
$$

and $F_{B}( \pm q \mid-q)=\sqrt{K}-F_{B}(\mp q \mid q)$; in addition,

$$
F_{B}( \pm q \mid \lambda) \approx c \sqrt{K} /(\pi k) \ll 1 \text { for } q, c \ll \lambda \approx k .
$$

Eq. (10) can be derived [20] from Ref. [22]. Eq. (11) follows from expansion of right hand side of Eq. (8) combined with $\rho( \pm q)=\sqrt{K} / 2 \pi$, see e.g. Eqs. (I.9.20-I.9.22) of Ref. [3].

We can calculate the exponents using the method of Refs. [6, 7, 8, 10]. For example, exponents at $\varepsilon_{1}(k)>0$ are evaluated using effective Hamiltonian $H=H_{0}+H_{d}+$ $H_{\text {int }}$, where

$$
H_{0}=\frac{v}{2 \pi} \int d x\left(K(\nabla \theta)^{2}+\frac{1}{K}(\nabla \phi)^{2}\right),
$$




$$
\begin{array}{r}
H_{d}=\int d x d^{\dagger}(x)\left(\varepsilon_{1}(k)-i \frac{\partial \varepsilon_{1}(k)}{\partial k} \frac{\partial}{\partial x}\right) d(x), \\
H_{\text {int }}=\int d x\left(V_{R} \nabla \frac{\theta-\phi}{2 \pi}-V_{L} \nabla \frac{\theta+\phi}{2 \pi}\right) d^{\dagger}(x) d(x) .
\end{array}
$$

Here $d^{\dagger}$ creates an extra particle with momentum near $k$, and we use the notations of Ref. [14], such that $\Psi_{B}(x, t) \sim e^{i \theta(x, t)},\left[\phi(x), \nabla \theta\left(x^{\prime}\right)\right]=i \pi \delta\left(x-x^{\prime}\right)$. The effective Hamiltonian, Eqs. (12)-(14), discriminates states created by $d^{\dagger}$ from low-lying states of the rest of the system which are described as LL. This separation is possible only in the investigation of response functions very close to the Lieb's modes, such as line $\varepsilon_{1}(k)$ in Fig. 2, and only due to the finite curvature of the underlying boson spectrum. Under these circumstances, excitations close to the edge can be distinguished from the excitations of the LL. Response functions evaluated from Eqs. (12)-(14) are valid in a narrow region of width vanishing as $\sim k^{2}$ at $k \rightarrow 0$; see discussion after Eq. (27).

Singular parts of DSF and spectral function are

$$
\begin{array}{r}
S(k, \omega) \sim \int d x d t e^{i \omega t}\left\langle d \Psi(x, t) \Psi d^{\dagger}(0,0)\right\rangle_{H_{0}+H_{d}+H_{i n t}}, \\
A(k, \omega) \sim \int d x d t e^{i \omega t}\left\langle d(x, t) d^{\dagger}(0,0)\right\rangle_{H_{0}+H_{d}+H_{i n t}} .
\end{array}
$$

The crucial step in our approach is the identification of the unitary transformation which removes an interaction term $H_{\text {int }}$ from the Bethe ansatz solution. This can be done by noticing that $H_{0}$ becomes a sum of noninteracting modes after transformation $\phi=\sqrt{K} \tilde{\phi}, \theta=\tilde{\theta} / \sqrt{K}$. If one refermionizes fields $\tilde{\phi}, \tilde{\theta}$, one obtains a noninteracting Luttinger model with two branches. Fermionic excitations of this model with momenta $k_{j}>0$ correspond to low-energy particlelike excitations of the Bethe ansatz with quasimomenta $\nu_{j}>q$, and $k_{j} \propto \nu_{j}-q$. The state where a $d$ particle is present corresponds to a state with occupied quasimomentum $\lambda$. For noninteracting fermions, phase shift on the particle $d$ can be written as $\delta(k)=2 \pi\left(k_{j}-\tilde{k}_{j}\right) /\left(k_{j+1}-k_{j}\right)$, where $\tilde{k}_{j}$ is the momentum of the new eigenstate in the presence of particle $d$. The same quantity in the Bethe ansatz solution can be calculated using Eq. (7), and by noticing that the shift of quasimomenta by $\nu_{j+1}-\nu_{j}$ corresponds to a phase shift $2 \pi$. Then interaction term $H_{\text {int }}$ can be removed [23, 24] by unitary transformation $U^{\dagger}\left(H_{0}+H_{d}+H_{\text {int }}\right) U$, where

$$
\begin{array}{r}
U^{\dagger}=e^{i \int d x\left(\frac{\delta_{+}}{2 \pi}(\tilde{\theta}(x)-\tilde{\phi}(x))-\frac{\delta_{-}}{2 \pi}(\tilde{\phi}(x)+\tilde{\theta}(x))\right) d^{\dagger}(x) d(x),} \\
\delta_{ \pm}=2 \pi F_{B}( \pm q, \lambda) .
\end{array}
$$

A standard calculation [14, 23, 24, 25] then leads to

$$
\begin{array}{r}
\mu_{1,2}=1-\frac{1}{2}\left(\frac{1}{\sqrt{K}}+\frac{\delta_{+}-\delta_{-}}{2 \pi}\right)^{2}-\frac{1}{2}\left(\frac{\delta_{+}+\delta_{-}}{2 \pi}\right)^{2}, \\
\overline{\mu_{ \pm}}=1-\frac{1}{2}\left(\frac{\delta_{+}-\delta_{-}}{2 \pi}\right)^{2}-\frac{1}{2}\left(\frac{\delta_{+}+\delta_{-}}{2 \pi}\right)^{2}
\end{array}
$$

Exponents $\underline{\mu_{+}}$and $\underline{\mu_{-}}$are more complicated. For example, $\mu_{+} \overline{\text { corresponds }}$ to a state with one additional particle, total momentum $k$, and the smallest possible energy $\varepsilon_{2}(k)$. Such state is given by two extra particles at the right quasifermi surface and a hole. To evaluate $\mu_{+}$, one needs to calculate the correlator $\left\langle d^{\dagger} \Psi \Psi(x, t) \Psi^{\dagger} \Psi^{\dagger} d(0,0)\right\rangle$, where $d$ creates a hole. One obtains

$$
\underline{\mu_{ \pm}}=1-\frac{1}{2}\left(\frac{2}{\sqrt{K}}+\frac{\delta_{+}-\delta_{-}}{2 \pi}\right)^{2}-\frac{1}{2}\left(\frac{\delta_{+}+\delta_{-}}{2 \pi}\right)^{2} .
$$

In Eqs.(16)-(18) one should choose $\lambda>q$ for $\mu_{1}, \overline{\mu_{+}}, \underline{\mu_{-}}$, and $|\lambda|<q$ for $\mu_{2}, \mu_{+}, \overline{\mu_{-}}$.

Using Eqs. (10)-(11), one can derive analytically the behavior of exponents near $k=0,2 \pi D$ and at $k \rightarrow \infty$. DSF exponents are given by

$$
\begin{aligned}
\mu_{1,2}(0) & =0, \\
\mu_{2}(2 \pi D-0)=2 \sqrt{K}-2 K & <0, \\
\mu_{1}(\infty)=1-1 /(2 K) & >0 .
\end{aligned}
$$

Exponents for the spectral function at low energies equal

$$
\begin{array}{r}
\overline{\mu_{ \pm}}(0)=1 / \sqrt{K}-1 /(2 K)>0, \\
\underline{\mu_{ \pm}}(0)=-1 / \sqrt{K}-1 /(2 K)<0, \\
\overline{\mu_{-}}(2 \pi D-0)=-2 K+2 \sqrt{K}+1 / \sqrt{K}-1 /(2 K), \\
\underline{\mu_{+}}(2 \pi D-0)=-2 K+2 \sqrt{K}-1 / \sqrt{K}-1 /(2 K),
\end{array}
$$

and for large momenta one has

$$
\underline{\mu_{-}}(\infty)=1-2 / K \text {. }
$$

For $k \rightarrow \infty$ Eq. (17) gives $\overline{\mu_{+}} \rightarrow 1$, which is a certain way to parameterize an expected result $A(k, \omega) \sim \delta\left(\omega-k^{2}\right)$, since $\delta\left(\omega-k^{2}\right) \sim \lim _{\overline{\mu_{+}} \rightarrow 1} \int d t e^{i\left(\omega-k^{2}\right) t} / t^{1-\overline{\mu_{+}}}$.

Numerical solutions show that all exponents are monotonic functions of $k$, lying between limiting values cited above. Exponents $\overline{\mu_{-}}$and $\mu_{-}$can change sign as a function of $k$. Exponent $\overline{\mu_{-}}$changes its sign from positive at $k=0$ to negative at $k=2 \pi D$ for sufficiently weak interactions, at $K>K_{c}=1 / 4+\sqrt{5} / 4+1 / 2 \sqrt{1 / 2+\sqrt{5} / 2} \approx$ 1.445 , and $\mu_{-}$can also change its sign from negative at $k=0$ to positive at $k=\infty$ for $K>2$. Our results are in full agreement with previously published limiting cases [8, 9] , and agree qualitatively with the numerical calculations of DSF [4] and spectral function [5].

One of the main achievements of the LL approximation [11, 12, 13, 14, 25] is the calculation of exponents in dynamic correlation functions in the limit $k \rightarrow 2 \pi D n$, where $n$ is any integer. We note however that exact exponents (20)-(25) in the immediate vicinity of the edges are different. They show markedly non-LL behavior, e.g. they can depend on $\sqrt{K}$, while LL exponents depend only on $K$ and $1 / K$. To illustrate the failure of LL exponents 
$\omega$

$\mathrm{A}(\mathrm{k}, \omega)$

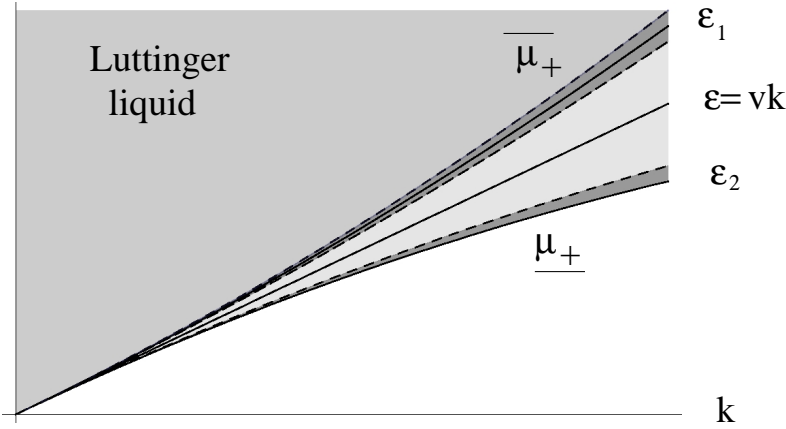

FIG. 2: Spectral function $A(k, \omega)$ for $\omega>0$ near $k=0$. Exact exponents $\overline{\mu_{+}}=1 / \sqrt{K}-1 /(2 K), \mu_{+}=-1 / \sqrt{K}-1 /(2 K)$ are valid only in the immediate vicinities of $\varepsilon_{1}$ and $\varepsilon_{2}$ marked by dashed lines. Luttinger liquid behavior given by Eqs. (27) is valid only sufficiently far away from $\varepsilon=v k$, for $\omega-v k \gg$ $\varepsilon_{1}-\varepsilon_{2}$.

in the immediate vicinity of the edge we consider $A(k, \omega)$ for $\omega>0$ near $k=0$ in more detail; see Fig. 2] LL theory assumes linear spectrum $\varepsilon(k) \approx v k$ and predicts [13, 14] at $k \ll v$

$$
A(k, \omega) \sim\left\{\begin{array}{c}
0, \text { if } \omega<v k, \\
(\omega-v k)^{1 /(4 K)-1} \text { if } 0<\omega-v k \ll \omega, \\
\omega^{1 /(2 K)-2} \text { if } v k \ll \omega .
\end{array}\right.
$$

Difference between the second and third lines here arises from the fact that only one branch contributes to the exponent for $0<\omega-v k \ll \omega$, while both left- and rightmovers contribute at $v k \ll \omega$. One should note however, that 0 in condition $0<\omega-v k \ll \omega$, is an artifact of the linear spectrum approximation, which breaks down at energy scales on the order of $\varepsilon_{1}(k)-\varepsilon_{2}(k) \sim k^{2}$. It is precisely within this region where exact results (22),(23) are applicable. Each of these exponents is valid in the vicinities of $\varepsilon_{1(2)}(k)$ much narrower than $\varepsilon_{1}(k)-\varepsilon_{2}(k) \sim k^{2}$. These exponents describe the response of the system at $k \rightarrow 0$ beyond linear hydrodynamic approximation. Nevertheless, for the Lieb-Liniger model they turn out to depend only on the LL parameter $K$, since phase shifts at quasifermi surface are also related to $K$; see Eq. (10). Far away from $k \rightarrow 2 \pi D n$, regions of validity of exponents (17), (18) widen, and the singularities constitute the main features of the spectral function.

To summarize, we have found exact exponents characterizing the singularities of the dynamic structure factor and spectral function along the dispersion curves of the collective modes of a 1D Bose gas. The found exponents are related to the known Bethe ansatz solution of the Lieb-Liniger model. Remarkably, the Luttinger liquid theory predictions for the exponents fail even at low energies, once the immediate vicinities of the edges are considered.

We thank A. Kamenev for useful discussions. This work was supported by NSF grants No. DMR-0749220 and No. DMR-0754613.

Note added. - After the essential part of this work has been completed, preprint to Ref. [26] has appeared, where equations similar to our Eqs. (8) and (16) were also reported for DSF of $1 \mathrm{D}$ fermions on a lattice.

[1] T. Kinoshita, T. Wenger, and D. S. Weiss, Nature 440, 900 (2006).

[2] E.H. Lieb and W. Liniger, Phys. Rev. 130, 1605 (1963); E.H. Lieb, ibid. 130, 1616 (1963).

[3] V.E. Korepin, N.M. Bogoliubov, and A.G. Izergin, Quantum Inverse Scattering Method and Correlation Functions (Cambridge University Press, Cambridge, 1993).

[4] J.-S. Caux and P. Calabrese, Phys. Rev. A 74, 031605(R) (2006).

[5] J.-S. Caux, P. Calabrese, and N.A. Slavnov, J. Stat. Mech. (2007) P01008. This work considered $A(k, \omega<0)$.

[6] M. Pustilnik, M. Khodas, A. Kamenev, and L.I. Glazman, Phys. Rev. Lett. 96, 196405 (2006).

[7] M. Khodas, M. Pustilnik, A. Kamenev, and L.I. Glazman, Phys. Rev. B 76, 155402 (2007).

[8] M. Khodas, M. Pustilnik, A. Kamenev, and L.I. Glazman, Phys. Rev. Lett. 99, 110405 (2007).

[9] M. Khodas, A. Kamenev, and L.I. Glazman, arXiv:0710.2910 1.

[10] R.G. Pereira, S.R. White, and I. Affleck, Phys. Rev. Lett. 100, 027206 (2008).

[11] K.B. Efetov and A.I. Larkin, Sov. Phys. JETP 42, 390 (1975) .

[12] F.D.M. Haldane, Phys. Rev. Lett. 47, 1840 (1981).

[13] M.A. Cazalilla, J. Phys. B 37, S1 (2004).

[14] T. Giamarchi, Quantum Physics in One Dimension (Clarendon, Oxford, 2004).

[15] U. Schollwöck, Rev. Mod. Phys. 77, 259 (2005).

[16] D.M. Stamper-Kurn et al., Phys. Rev. Lett. 83, 2876 (1999).

[17] L.-M. Duan, Phys. Rev. Lett. 96, 103201 (2006).

[18] T.-L. Dao, A. Georges, J. Dalibard, C. Salomon, and I. Carusotto, Phys. Rev. Lett. 98, 240402 (2007).

[19] A.A. Abrikosov, L.P. Gorkov, and I.E. Dzyaloshinski, Methods of Quantum Field Theory in Statistical Physics (Dover, New York, 1963).

[20] Shift function $F(\nu \mid \lambda)$ defined by Eq. (I.4.25) of Ref. 3] is related to ours as $F(\nu \mid \lambda)=F_{B}(\nu \mid \lambda)-\pi \rho(\nu)$. Ref. [3] assumes a change of periodic boundary conditions to antiperiodic ones with the change of particle number (see p.23), and phase shifts calculated there correspond to the fermionic Cheon-Shigehara model [21] dual to the bosonic Lieb-Liniger model.

[21] T. Cheon and T. Shigehara, Phys. Rev. Lett. 82, 2536 (1999).

[22] V. Korepin and N. Slavnov, Eur. Phys. J. B 5, 555 (1998).

[23] K.D. Schotte and U. Schotte, Phys. Rev. 182, 479 (1969).

[24] L. Balents, Phys. Rev. B 61, 4429 (2000).

[25] A. Gogolin, A. Nersesyan, and A. Tsvelik, Bosonization and Strongly Correlated Systems (Cambridge University Press, Cambridge, 1998).

[26] V.V. Cheianov and M. Pustilnik, Phys. Rev. Lett. 100, 126403 (2008). 